\title{
SOME RING THEORETIC SCHRÖDER-BERNSTEIN THEOREMS
}

\author{
BY \\ IAN G. CONNELL
}

1. Introduction. If $a$ and $b$ are objects in a category let us write $a \leqq b$ to indicate that there is a map from $a$ to $b$, and $a \sim b$ to indicate that there is an equivalence (i.e., invertible map) between $a$ and $b$. The question then arises whether the category has the Schröder-Bernstein property, viz:

$$
a \leqq b \text { and } b \leqq a \Rightarrow a \sim b .
$$

(The classical Schröder-Bernstein theorem refers to the category of sets and faithful mappings; in this category an equivalence is a faithful onto mapping.)

The case we investigate here is the following. Let $R$ be a ring and $A$ an $R$-module. (All rings are supposed to be associative with 1 and all modules right unitary modules.) The objects of the category are the elements of $A$ and the maps are pairs $(a, x)$ where $a \in A, x \in R$. The product $(a, x)(b, y)$ is defined if and only if $b=a x$ and then is $(a, x y)$. Thus $(a, x)$ is a map from $a$ to $a x$, the maps of the form $(a, 1)$ are the identities, and the equivalences are maps of the form $(a, u)$ where $u \in R^{*}$, the group of units of $R$.

The property in question is simply: if $a$ and $b$ are elements of $A$ generating the same submodule (that is, there exist $x, y \in R$ such that $a x=b$ and $b y=a$ ) then there exists a unit $u \in R^{*}$ such that $a u=b$. If $A$ has this property we say that $A$ is $P_{1}$.

It will be convenient to extend this notion. The direct sum $A^{n}$ of $n$ copies of $A$ is canonically a module over the ring $R_{(n)}$ of $n$ by $n$ matrices, and we say that $A$ is $P_{n}$ if $A^{n}$ is $P_{1}$ as a module over $R_{(n)}$. It is easily seen that this is equivalent to the following condition on $A$ : if the two sets $\left\{a_{1}, \ldots, a_{n}\right\}$ and $\left\{b_{1}, \ldots, b_{n}\right\}$ generate the same $R$-submodule of $A$ then there exists an invertible matrix $U \in R_{(n)}^{*}$ such that $\left(a_{1}, \ldots, a_{n}\right) U=\left(b_{1}, \ldots, b_{n}\right)$. (The order chosen for the $a_{i}$ and $b_{i}$ does not matter since permutation matrices are invertible.)

If $A$ is $P_{n}$ for all $n$ we say that $A$ is $P_{\infty}$; and if all $R$-modules are $P_{n}$ or $P_{\infty}$ we say that $R$ is $\pi_{n}$ or $\pi_{\infty}$, respectively. When we say that $R$ is $P_{n}$ we are referring to $R$ as a module over itself.

Further notation: $\Re(R)$ denotes the Jacobson radical of the ring $R ; \prod R_{i}$ denotes the complete direct product of the rings $R_{i} ; \oplus A_{i}$ denotes the direct sum of the modules $A_{i}$. When we say that $R$ is artinian or noetherian we are referring to right ideals (since we insist that all modules be right modules). By integral domain, or simply domain, we understand a commutative ring without zero divisors.

Received by the editors March 10, 1967. 


\section{Preliminaries.}

Proposition 1. $R$ is $\pi_{1}$ if and only if for each pair $x, y \in R$ there exists an $r \in R$ such that $x+(1-x y) r \in R^{*}$.

Proof $\left({ }^{1}\right)$. If $R$ is $\pi_{1}$ then the $R$-module $R / J$ is $P_{1}$, where $J=(1-x y) R$. Now $x y \equiv 1 \bmod J$ so $1+J$ and $x+J$ both generate $R / J$, hence there exists a unit $u$ such that $(1+J) u=x+J$, i.e., $u=x+(1-x y) r$ for some $r$.

Conversely let $A$ be an $R$-module, $a, b \in A$ and $x, y \in R$ such that $a x=b, b y$ $=a$. If $x+(1-x y) r=u \in R^{*}$ then $a u=b$, which shows that $A$ is $P_{1}$ and, since $A$ is arbitrary, that $R$ is $\pi_{1}$.

This criterion will usually be applied in the form: if $x y+z=1$ then there exists an $r$ such that $x+z r \in R^{*}$.

Proposition 2. For any positive integers $n$ and $k, R_{(n)}$ is $\pi_{k}$ if and only if $R_{(k)}$ is $\pi_{n}$.

By the isomorphism $R_{(n)(k)} \cong R_{(n k)}$ this clearly results from the following special case.

CoROllary 1. $R$ is $\pi_{n}$ if and only if $R_{(n)}$ is $\pi_{1}$.

Proof. If $R_{(n)}$ is $\pi_{1}$ it follows by definition that $R$ is $\pi_{n}$. Conversely let $R$ be $\pi_{n}$ and $x y+z=1$ an equation in $R_{(n)}$. The columns of $z$ regarded as elements of the free $R$-module $R^{n}$ generate a submodule $M$ and the above equation states that the columns of $x \bmod M$ generate the $R$-module $R^{n} / M$. But the columns of the unit matrix 1 taken $\bmod M$ also generate $R^{n} / M$, and since $R$ is $\pi_{n}$ there exists $u \in R_{(n)}^{*}$ such that $1 u \equiv x \bmod M$, i.e., $u=x+z t$ for some $t \in R_{(n)}$ and therefore $R_{(n)}$ is $\pi_{1}$.

COROLlaRY 2. If $R$ is $\pi_{\infty}$ then so is $R_{(n)}$ for each $n$.

COROLlARY 3. If the rings $R_{i}$ are all $\pi_{n}$, or $\pi_{\infty}$, then so is $R=\prod R_{i}$.

For $R_{(n)}=\prod R_{i(n)}$, so it is enough to consider the case $n=1$, which is clear since $R^{*}=\prod R_{i}^{*}\left(^{2}\right)$.

Proposition 3. If $R$ is $\pi_{n}$ then $R$ is $\pi_{k}$ for each divisor $k$ of $n$; in particular, $R$ is $\pi_{1}$.

( $\left.{ }^{1}\right)$ This characterization can be stated in a more symmetrical, though not so convenient, form as follows: Let $R_{s}$ denote the set of $(x, y) \in R \times R$ for which there exists an $r$ such that $x r+y \in R^{*}$ and $R_{d}$ the set of $(x, y)$ for which there exists an $r$ such that $x+y r \in R^{*}$. Then $R$ is $\pi_{1}$ if and only if $R_{s}=R_{d}$. Another characterization is given in Proposition 14 below.

${ }^{2}$ ) This result is also obvious from the point of view of universal algebra: the class of $\pi_{n}$ rings, or $\pi_{\infty}$ rings, is equationally defined. For example when $n=1$ we have besides the usual ring operations and axioms of equational type two more binary operations $f(x, y)$ and $g(x, y)$ and the additional equations $[x+(1-x y) f(x, y)] g(x, y)=g(x, y)[x+(1-x y) f(x, y)]=1$. Hence direct products exist in this category and are constructed in the usual cartesian way. For the $\pi_{n}$ property one needs a number of $2 n^{2}$-ary operations with appropriate equations; and for the $\pi_{\infty}$ property all these operations and equations are adjoined. 
Proof. By Corollary 1 above, it is sufficient to deal with the case $k=1$; for then in general, if $n=k m$ and $R$ is $\pi_{n}$ then $R_{(n)}=R_{(k)(m)}$ is $\pi_{1}$ whence $R_{(k)}$ is $\pi_{m}$, therefore $\pi_{1}$, and $R$ is $\pi_{k}$. Thus consider $x y+z=1, x, y, z \in R$, and form $X=\operatorname{diag}(x, I)$, $Y=\operatorname{diag}(y, I), Z=\operatorname{diag}(z, 0) \in R_{(n)}$ where $I$ and 0 denote the $n-1$ by $n-1$ identity and zero matrices respectively. Since $R_{(n)}$ is $\pi_{1}$ there exists $W \in R_{(n)}$ such that $X+Z W \in R_{(n)}^{*}$. This gives $x+z w \in R^{*}$, where $w$ is the top left entry in $W$, as required.

This proposition cannot be generalized to the $P_{n}$ property; for example, the cyclic group of order 5 , as a $Z$-module, is not $P_{1}$ but is $P_{n}$ for all $n \geqq 2$ (see $\S 6$ below).

Proposition 4. If $R / \Re(R)$ is $\pi_{n}$, or $\pi_{\infty}$, then so is $R$.

Proof. Since $\Re\left(R_{(n)}\right)=\Re(R)_{(n)}\left({ }^{3}\right)$ and $R_{(n)} / \Re\left(R_{(n)}\right)=[R / \Re(R)]_{(n)}$, by Proposition 2, Corollary 1 , it is sufficient to consider the case $n=1$. Suppose then $x y+z=1$, $x, y, z \in R$, and therefore $\bar{x} \bar{y}+\bar{z}=\overline{1}$, letting the bar indicate images in $\bar{R}=R / \Re(R)$. By assumption $\bar{x}+\bar{z} \bar{r}=\bar{u} \in \bar{R}^{*}$ for some $\bar{r} \in \bar{R}$, so $x+z r=u+j, j \in \Re(R)$. Now if $\bar{u} \bar{v}=\bar{v} \bar{u}=\overline{1}$, then $(u+j) v=1+k, k \in \Re(R)$; but then $1+k \in R^{*}$ so $u+j$ has a right inverse, similarly a left inverse, and $u+j \in R^{*}$ as required.

If $R$ is $\pi_{n}$ and $J$ is any ideal then $R / J$ is $\pi_{n}$. (This follows from the original definition of $\pi_{n}$ by giving each $R / J$-module its canonical structure as an $R$-module.) Thus

COROLlaRY. If $J \subseteq \Re(R)$ then $R / J$ is $\pi_{n}\left(\pi_{\infty}\right)$ if and only if $R$ is $\pi_{n}\left(\pi_{\infty}\right)$.

Proposition 5. Let the rings $R_{i}$ form a direct system and let $R$ be their injective limit. If the $R_{i}$ are $\pi_{n}$, or $\pi_{\infty}$, then so is $R\left(^{4}\right)$.

Proof. The process of forming matrix rings commutes with the injective limit operation, so we can restrict our attention to the case $n=1$. If $x, y \in R$ there exists an $R_{i}$ and $x^{\prime}, y^{\prime} \in R_{i}$ such that $x^{\prime} \sigma_{i}=x, y^{\prime} \sigma_{i}=y$, where $\sigma_{i}: R_{i} \rightarrow R$ is the canonical homomorphism. Since $R_{i}$ is $\pi_{1}$ there exist $r^{\prime} \in R_{i}, u^{\prime} \in R_{i}^{*}$ such that $u^{\prime}=x^{\prime}+$ $\left(1-x^{\prime} y^{\prime}\right) r^{\prime}$. Applying $\sigma_{i}$ gives the result.

The following corollary is useful for getting examples. Note that a subring always contains the unit element of the over-ring.

$\left({ }^{3}\right)$ This well-known formula is a corollary of Proposition A in the Appendix.

$\left.{ }^{4}\right)$ The analogous statement for projective limits is true in particular cases, e.g., in the case of direct products, as we have seen. As another example, take any ring $R$ and consider the factor rings $R / I$ which are artinian (in exceptional cases this collection is vacuous). These rings canonically form an inverse system and by Theorem 1 below and an argument using linear compactness (cf. [8]) their projective limit $\hat{R}$ is $\pi_{\infty}$. Of course in the equationally defined category of $\pi_{n}$ (or $\pi_{\infty}$ ) rings there is no problem; the point of this proposition is that the homomorphisms defining the direct system need not respect the additional operations as described in the footnote to Corollary 3, Proposition 2. 
COROLlaRY. If each finitely generated subring of $R$ is contained in some $\pi_{n}\left(\pi_{\infty}\right)$ subring $S$ of $R$, then $R$ is $\pi_{n}\left(\pi_{\infty}\right)$.

For $R$ is the injective limit of the various $S$.

We omit the straightforward proofs of the next two results.

Proposition 6. Let $A_{i}$ form a direct system of $R$-modules and let $A=\operatorname{Lim}_{\rightarrow} A_{i}$. If the $A_{i}$ are $P_{n}$ or $P_{\infty}$ then so is $A$.

Proposition 7. If the $R$-module $A$ is $P_{\infty}$ then for an arbitrary cardinal $I, A^{I}$ is $P_{\infty}$ as $R_{(I)}$-module, where $R_{(I)}$ denotes the ring of row-finite I by I matrices.

3. $\pi_{\infty}$ rings.

THEOREM 1. Let $R$ be $a$ ring and $A$ an $R$-module of finite length. Then the commutator $\operatorname{End}_{R} A$ is $\pi_{\infty}$.

Proof. Let $S=\operatorname{End}_{R} A$. By Proposition 4 it is sufficient to prove that $T=S / \Re(S)$ is $\pi_{\infty}$. By Proposition B in the Appendix, $T$ is a product of matrix rings $E_{(n)}$ over skew-fields $E$ and by Corollary 3 to Proposition 2 it is sufficient to prove that $E_{(n)}$ is $\pi_{\infty}$. (In effect we have reduced the theorem to the case where $R$ is a skewfield.) By Proposition 2 and the formula $E_{(n)(m)}=E_{(n m)}$, it is sufficient to prove that $E_{(n)}$ is $\pi_{1}$.

Referring to Proposition 1, we thus assume that $x y+z=1, x, y, z \in E_{(n)}$. This equation implies that the (right) column space $C(x)$ of $x$ together with the column space $C(z)$ of $z$ include a basis for $n$-dimensional space $V$ over $E$. Numbering the columns $x_{i}$ of $x$ and the columns $z_{i}$ of $z$ appropriately, let $x_{1}, \ldots, x_{k}$ be a basis for $C(x)$ and $x_{1}, \ldots, x_{k}, z_{k+1}, \ldots, z_{n}$ a basis for $V$. We define the matrix $u$ by numbering its columns the same way as was done for $x$ and putting $u_{i}=x_{i}$ for $1 \leqq i \leqq k$ and $u_{i}=x_{i}+z_{i}$ for $k<i \leqq n$. Thus $u=x+z r$ for some $r$. Finally $u$ is invertible since $C(u)$ includes the above basis for $V$.

As a special case we have the following solution to a problem posed by B. Brown.

COROLlary 1. If $a$ and $b$ are elements of the finite abelian group $A$ and $\alpha$ and $\beta$ are endomorphisms such that $a \alpha=b, b \beta=a$, then there exists an automorphism $u$ such that $a u=b$.

In $\S 5$ we consider other classes of abelian groups for which this result holds.

The results of the preceding section immediately yield

COROLLARY 2. If $R / \Re(R)$ is a direct product $\prod E_{i\left(n_{i}\right)}$ of (arbitrarily many) matrix rings over skew-fields, then $R$ is $\pi_{\infty}$.

This class of rings includes all semiperfect rings, the latter class including all artinian and all local rings (cf. [5]). Zelinsky [8] has characterized the rings $\prod E_{i\left(n_{i}\right)}$ in terms of linear compactness. 
Call $A$ finite dimensional (after Goldie) if it does not contain an infinite family of submodules whose sum is direct. Secondly, we call $A$ selfinjective $\left({ }^{5}\right)$ if every partial endomorphism of $A$ can be extended to a full endomorphism; that is, if $B$ is a submodule and $\alpha \in \operatorname{Hom}_{R}(B, A)$ then there exists $\beta \in \operatorname{End}_{R} A$ which agrees with $\alpha$ on $B$. Clearly every injective module is selfinjective.

Corollary 3. If $A$ is finite dimensional selfinjective then $\operatorname{End}_{R} A$ is $\pi_{\infty}$.

For $\operatorname{End}_{R} A$ is semiperfect. (See [5] where a proof is given when $A$ is finite dimensional injective; as indicated there, the same proof works in the more general case.)

The next proposition is useful in getting further classes of $\pi_{\infty}$ rings.

Proposition 8. Let $A$ and $B$ be $R$-modules such that $\operatorname{Hom}_{R}(A, B)=0$ and $R_{1}=\operatorname{End}_{R} A$ and $R_{2}=\operatorname{End}_{R} B$ are $\pi_{\infty}$. Then $R_{3}=\operatorname{End}_{R}(A \oplus B)$ is $\pi_{\infty}$.

Putting $\bar{R}_{i}=R_{i} / \Re\left(R_{i}\right)$, by Proposition A in the Appendix we have $\bar{R}_{3}=\bar{R}_{1} \times \bar{R}_{2}$, and therefore $R_{3}$ is $\pi_{\infty}$.

We conclude this section with some examples.

1. Every boolean ring is $\pi_{\infty}$. For every finitely generated subring is finite, hence $\pi_{\infty}$, and the Corollary to Proposition 5 applies $\left({ }^{6}\right)$. (This does not generalize to regular =absolutely flat rings; see example 3 below.) More generally, $R$ is $\pi_{\infty}$ if for every $x \in R$ there exists $n=n(x)>1$ such that $x^{n}=x$; for such an $R$ is commutative [2, p. 217], of finite characteristic, and the same argument applies.

2. If $R$ is semilocal, i.e., has only finitely many maximal right ideals $M_{1}, \ldots, M_{n}$ then $R$ is $\pi_{\infty}$. For each $R / M_{i}$ is an artinian $R$-module, hence so is $R / \Re(R) \subset R / M_{1} \oplus$ $\cdots \oplus R / M_{n}$, and therefore $R / \Re(R)$ is an artinian ring. (It can be shown that $R$ has only finitely many maximal left ideals so there is no need to specify 'right' semilocal.)

3. Call $R$ symmetric if $x y=1$ implies $y x=1$. (Since $R$ can be regarded as a subring of $R_{(n)}$, if $R_{(n)}$ is symmetric so is $R$; P. M. Cohn informs me that the converse, a question raised in [4, p. 466], is false.) If $R$ is $P_{1}$ then $R$ is symmetric; for $x y=1$ entails $x R=1 R$ so there is a unit $u$ such that $x=1 u$, whence $y=x^{-1}$. Now if $I$ is an infinite cardinal then the ring $R_{(I)}$ of row-finite $I$ by $I$ matrices is not symmetric, thus certainly not $\pi_{1}$ (and therefore not $\pi_{n}$ for any $n$ ).

For example if $F$ is a field and $A=F^{I}$ is the vector space of dimension $I$, then $F_{(I)}=\operatorname{End}_{F} A$ is not $\pi_{1}$. Note however, that $F_{(I)}$ is a regular ring. Also it is obvious that the ring of integers $Z=\operatorname{End}_{z} Z$ is not $\pi_{1}$ (cf. §6). These examples show that the assumption of finite length cannot be dropped from Theorem 1 and that neither assumption on $A$ can be dropped from Corollary 3.

(5) Johnson and Wong [3] call such a module quasi-injective. We feel that our terminology is more suggestive; it also has the advantage that when applied to the $R$-module $R$ it coincides with the usual notion of a (right) selfinjective ring.

$\left({ }^{6}\right)$ The fact that a boolean ring is $\pi_{1}$ is immediate from the identity $x+(1+x y)(1+x)=1$. 
In both these cases it happens that the module is $P_{\infty}$ with respect to the endomorphism ring (by Proposition 7 or 11, and Theorem 2); but even this is not a general rule. To see this it suffices to take $A=R$ a commutative $\left({ }^{7}\right)$ ring which is not $P_{1}$. An example is the ring of real continuous functions (see Kaplansky [4, p. 466]). It follows that the noetherian $\operatorname{ring} Z[x, y, z, t] / J$, where $J$ is the ideal generated by $x z-y$ and $y t-x$, is not $P_{1}$; for if this ring were $P_{1}$ it would follow easily that all commutative rings are $P_{1}$. Kaplansky [ibid.] gives another noetherian ring which is not $P_{1}$.

4. We now give two examples of subrings of $R_{(I)}$ which are $\pi_{\infty}$ when $R$ is $\pi_{\infty}$. For integers $n$ and $k$ we have the ring embedding $R_{(n)} \rightarrow R_{(n k)}$ given by $x \rightarrow$ $\operatorname{diag}(x, \ldots, x)$, and $R, R_{(2)}, R_{(3)}, \ldots$ thus forms a direct system of rings, the directed set being the natural numbers $N$ ordered by divisibility. By Proposition 5 , $\operatorname{Lim}_{\rightarrow} R_{(n)}$, the subring of 'periodic' matrices of $R_{(N)}$, is $\pi_{\infty}$. Secondly, the subring $T$ of $R_{(I)}$ consisting of those matrices which, apart from finitely many entries, are zero off the diagonal and have constant diagonal entry is $\pi_{\infty}$. For by taking $k$ a sufficiently large integer we see that a finitely generated subring of $T$ is contained in a subring isomorphic to $R_{(k)} \times R$ which is $\pi_{\infty}$, and the corollary to Proposition 5 applies. When $I$ is countable the elements of $T$ are matrices of the form $\operatorname{diag}(X, d, d, \ldots)$ where $X$ is an arbitrary finite square matrix and $d \in R$. Taking $R$ a field we thus obtain examples of nonnoetherian primitive rings which are $\pi_{\infty}[2$, p. 36].

5. If $S$ is a commutative ring and $R=S[x]$ is the polynomial ring over $S$ then $R$ is not $\pi_{1}$. For $1=(1-x)(1+x)+x^{2}$ and the criterion of Proposition 1 cannot be satisfied. Similarly, the primitive ring of [2, p. 22, Example 3(a)] and the noetherian simple ring of $\left[1\right.$, p. 60 , Exercise 13] are not $\pi_{1}$.

6. In $\S 6$ we will prove that the ring of algebraic integers in a finite extension of the rational field is not $\pi_{1}$, but the integral domain of all algebraic integers is $\pi_{1}$.

4. Dedekind domains. If $R$ is any ring we say the $R$-module $A$ is torsion-free if every finitely generated submodule is embeddable in a free module. (Of course these free modules can be taken to be finitely generated). Thus every projective module is torsion-free. When $R$ is an integral domain this coincides with the usual notion of torsion-free.

The following nontrivial result is due to Steinitz [6, II, p. 340].

THEOREM 2. Let $R$ be a dedekind domain and $n \geqq 1$. Then every torsion-free $R_{(n)}$-module is $P_{\infty}$.

Actually Steinitz proves the following.

THEOREM 2 '. If $a$ and $b$ are $m$ by $n$ matrices over the dedekind domain $R$ for which there exist $x, y \in R_{(n)}$ such that $a x=b, b y=a$ then there exists $u \in R_{(n)}^{*}$ such that $a u=b$.

${ }^{(7)} \mathrm{End}_{R} R=R^{\circ}$, the opposite ring of $R$, rather than $R$ since we insist that all module action occur on the right. We take $R$ commutative to avoid this trifling complication. 
We do not reproduce Steinitz's argument but prove the equivalence of the two theorems $\left({ }^{8}\right)$. Assuming the first theorem, let $a x=b, b y=a$ in the notation of the second. Adjoining an appropriate number of zero rows to the bottom of $a$ and $b$ we obtain $m^{\prime}$ by $n$ matrices $a^{\prime}$ and $b^{\prime}$ such that $a^{\prime} x=b^{\prime}, b^{\prime} y=a^{\prime}$ where $m^{\prime}$ is a multiple of $n$, say $m^{\prime}=k n$. Now $a^{\prime}$ and $b^{\prime}$ can be regarded as column vectors with entries from $R_{(n)}$, that is, elements of the free $R_{(n)}$-module $R_{(n)}^{k}$. But since this module is $P_{1}$ there exists $u \in R_{(n)}^{*}$ such that $a^{\prime} u=b^{\prime}$. Removing the superfluous zero rows we have $a u=b$, as required.

Conversely let $a_{1}, \ldots, a_{t}, b_{1}, \ldots, b_{t}$ be elements of the torsion-free $R_{(n)}$-module $A$ such that $a_{1} R_{(n)}+\cdots+a_{t} R_{(n)}=b_{1} R_{(n)}+\cdots+b_{t} R_{(n)}=B$, say. We embed $B$ in a free $R_{(n)}$-module, say of rank $m$. In terms of a basis each $a_{i}$ gives rise to a column vector of length $m$ with entries from $R_{(n)}$ and in this way $a_{1}, \ldots, a_{t}$ give rise to an $m$ by $t$ matrix $a$ over $R_{(n)}$ or, what is the same thing, an $m n$ by $t n$ matrix over $R$. Similarly the $b_{i}$ give rise to an $m n$ by $t n$ matrix $b$ and the assumption clearly amounts to $a x=b, b y=a$ for some $x, y \in R_{(t n)}$. By the second theorem there exists $u \in R_{(t n)}^{*}$ such that $a u=b$, and this is just the statement that $A$ is $P_{t}$ as $R_{(n)}$-module.

A particular case of this theorem is worth singling out: Theorem 53 of Hilbert's Zahlbericht, due to Hurwitz, which is the statement that $R$ is $P_{2}$. As is well known, every ideal of $R$ can be generated by a pair of elements and Theorem 53 says that any two pairs generating the same ideal are related by an invertible two by two matrix of $R_{(2)}$. As Hilbert points out, this allows one to give a purely arithmetical definition of the class number.

We now quote some related results of Kaplansky [4]. A right bézout ring is a ring $R$ in which every finitely generated right ideal is principal, and a right hermite ring is one with the property that for every $a, b \in R$ there exist $d \in R, u \in R_{(2)}^{*}$ such that $(a, b) u=(d, 0)$ (i.e., 1 by 2 matrices can be diagonalized). Now a right hermite ring is precisely a $P_{2}$ right bézout ring; the only nonobvious fact is that a right hermite ring must be $P_{2}$, and this is contained in the following.

PROPOSITION 9. If $R$ is a right hermite ring and $F$ is a free $R$-module of rank $m$ then $F$ is $P_{n}$ for every $n \geqq 2 m$; in particular $R$ is $P_{n}$ for all $n \geqq 2$.

Proof. Let $a_{1}, \ldots, a_{n}, b_{1}, \ldots, b_{n} \in F$ with $a_{1} R+\cdots+a_{n} R=b_{1} R+\cdots+b_{n} R=A$. As above, in terms of a basis the elements $a_{1}, \ldots, a_{n}$ give rise to an $m$ by $n$ matrix $a$ and by Theorem 3.5 of [4] there exists $u \in R_{(n)}^{*}$ such that $a u$ is triangular, say $a u=(d, 0)$ where $d \in R_{(m)}$ (with zeros above the diagonal) and 0 denotes the $m$ by $n-m 0$-matrix. Similarly for the $b_{i}$ we have $b v=\left(d^{\prime}, 0\right)$. Now the columns of $d$, regarded as elements of $F$, generate $A$, as do the columns of $d^{\prime}$. Hence there exist $x, y \in R_{(m)}$ such that $d x=d^{\prime}, d^{\prime} y=d$, and by Kaplansky's device [4, $\left.\S 4\right]$ there exists

$\left.{ }^{8}\right)$ Incidentally, by 'Grundmodul' Steinitz means a pure submodule of a finitely generated free module; surely this must constitute one of the earliest uses of the concept of purity. (Of course in the dedekind case a Grundmodul is the same thing as a direct summand of the free module.) 
$w \in R_{(2 m)}^{*}$ such that $(d, 0) w=\left(d^{\prime}, 0\right)$, where 0 now denotes the $m$ by $m 0$-matrix. Finally, if $z=\operatorname{diag}(w, I)$ where $I$ denotes the $n-2 m$ rowed identity matrix we have $\left(a_{1}, \ldots\right) u z v^{-1}=\left(b_{1}, \ldots\right)$ with $u z v^{-1} \in R_{(n)}^{*}$, as required.

This result can be substantially improved when 0-divisors are disallowed:

Proposition 10. Let $R$ be a right hermite ring without 0 -divisors and let $n \geqq 1$. Then every torsion-free $R_{(n)}$-module is $P_{\infty}$; in particular $R$ is $P_{\infty}$.

It is shown exactly as in the case of Theorem 2 that this is equivalent to what Kaplansky actually proves: if $a$ and $b$ are $m$ by $n$ matrices over $R$ such that $a x=b$, $b y=a$ for some $x, y \in R_{(n)}$, then there exists $u \in R_{(n)}^{*}$ such that $a u=b$.

In the commutative case it is easily seen that the $P_{2}$ property is automatic; that is, a bézout domain is the same thing as an hermite domain. In this case the following well-known result is an easy consequence.

COROLlARY. If $R$ is a bézout domain (in particular, a principal ideal domain) and the elements $a_{1}, \ldots, a_{n}, n \geqq 2$, generate the ideal $d R$ then $\left(a_{1}, \ldots, a_{n}\right)$ occurs as the first row of an $n$ by $n$ matrix with determinant $d$.

5. Commutation. Here we take up the question of when the $R$-module $A$ is $P_{\infty}$ as a module over its commutator $\Omega=\Omega(A)=\operatorname{End}_{R} A$. (We use the abbreviated notation $\Omega$ and $\Omega(A)$ when it is not ambiguous.) We have already seen that the answer is affirmative when $A$ has finite $R$-length, or when $A$ is finite dimensional selfinjective; indeed in these cases $\Omega$ is $\pi_{\infty}$, which is much more than is needed.

LEMMA 1. If $C$ is a class of $R$-modules closed under finite direct sums, then to show that each $A \in C$ is $P_{\infty}$ as $\Omega$-module it is sufficient to prove the $P_{1}$ property; and when this is the case, an arbitrary sum of members of $C$ is $P_{\infty}$ as $\Omega$-module.

The first statement is clear since $A^{n} \in C$ and $\Omega\left(A^{n}\right)=\Omega(A)_{(n)}$; the second statement follows from the first statement and the next lemma.

LeMma 2. If every pair of elements of $A$ is contained in an $R$-direct summand $B$ of $A$ which is $P_{1}$ as $\Omega(B)$-module, then $A$ is $P_{1}$ as $\Omega(A)$-module.

Proof. If $a \alpha=b, b \beta=a$ where $a, b \in A$, and $\alpha, \beta \in \Omega(A)$, let $A=B \oplus C$ be an $R$-direct decomposition with $i: B \rightarrow A, \pi: A \rightarrow B$ the canonical maps such that $a \pi, b \pi \in B$. Then $(a \pi) i \alpha \pi=b \pi,(b \pi) i \beta \pi=a \pi$ where $i \alpha \pi$ and $i \beta \pi \in \Omega(B)$, so there exists $\gamma \in \Omega(B)^{*}$ such that $a \pi \gamma=b \pi . \gamma \oplus 1$ is the required automorphism of $A$.

Lemma 3. Let $A$ be an $R$-module and I its $R$-injective hull. Then $A$ is $R$-selfinjective if and only if $A$ is an $\Omega(I)$-submodule of $I$.

For the proof see [3].

Proposition 11. Let $R$ be noetherian and $A$ R-selfinjective. Then $A$ is $P_{\infty}$ as $\Omega(A)$-module.

Proof. First let $A$ be injective. By Lemma 1 we must show that $A$ is $P_{1}$. If $a, b \in A$ let $B=a R+b R$ and let $B \subseteq I \subseteq A$ where $I$ is an injective hull of $B . I$ is a 
direct summand of $A$ and by Lemma 2 we wish to prove that $I$ is $P_{1}$ as $\Omega(I)$ module. But $B$, being a noetherian module, is finite dimensional, hence so is $I$ since $I$ is an essential extension of $B$ (i.e., if $X$ is a nonzero submodule of $I$ then $B \cap X \neq 0)$. The result in this case now follows from Corollary 3 of Theorem 1 .

Now let $A$ be selfinjective, $I$ its injective hull and $\left(a_{1}, \ldots, a_{n}\right) \alpha=\left(b_{1}, \ldots, b_{n}\right)$, $\left(b_{1}, \ldots, b_{n}\right) \beta=\left(a_{1}, \ldots, a_{n}\right)$ where $a_{i}, b_{i} \in A, \alpha=\left(\alpha_{i j}\right), \beta=\left(\beta_{i j}\right) \in \Omega(A)_{(n)}$. Each $\alpha_{i j}$, $\beta_{i j}$ can be extended to an $R$-endomorphism of $I$ and by the injective case there exists a $\gamma \in \Omega(I)_{(n)}^{*}$ such that $\left(a_{1}, \ldots\right) \gamma=\left(b_{1}, \ldots\right)$. The result follows from Lemma 3 by restricting the $\gamma_{i j}$ to $A$.

The condition that $R$ be noetherian cannot be dropped. For example take $A=R=$ the ring of (say) countable row-finite matrices over a field. This ring is selfinjective [7, Theorem 5], i.e., $A$ is $R$-selfinjective. But $A$ is not $P_{1}$ as $\Omega(A)$-module (cf. example 3 of $\S 3$ ).

If $R$ is an integral domain and $A$ is a torsion-free divisible (=torsion-free injective) $R$-module then $A$ is $P_{1}$, hence (by Lemma 1) $P_{\infty}$ as $\Omega$-module. For $A$ is a vector space over the quotient field $K$ of $R, \Omega_{R}(A)=\Omega_{K}(A)$, and there is an automorphism of $A$ taking any nonzero element $a$ onto any other nonzero element $b$. This remark obviously has generalizations, for example to the case where $R$ is a commutative ring whose classical ring of quotients is noetherian.

We omit the trivial proofs of the next two lemmas.

Lemma 4. If each $A_{i}$ is $P_{1}$ as an $R_{i}$-module then $\oplus A_{i}$ as a $\prod R_{i}$-module with component-wise action is $P_{1}$.

LEMMA 5. Let $R$ be commutative (so $R$ can be identified with its opposite ring $R^{\circ}$ ). If the free module $A=R^{n}$ is $P_{n}$ as $R$-module then $A$ is $P_{1}$ as $\Omega$-module.

The next result is a variant of Proposition 8.

Lemma 6. If $A=B \oplus C$ is an $R$-direct sum such that $\Omega(B)$ is $\pi_{1}, C$ is $P_{1}$ as $\Omega(C)$ module, and $\operatorname{Hom}_{R}(B, C)=0$, then $A$ is $P_{1}$ as $\Omega(A)$-module.

Proof. The elements of $\Omega(A)$ are of the form

$$
x=\left(\begin{array}{ll}
\alpha & 0 \\
\beta & \gamma
\end{array}\right), \quad \alpha \in \Omega(B), \quad \beta \in \operatorname{Hom}_{R}(C, B), \quad \gamma \in \Omega(C),
$$

the units being those $x$ with $\alpha \in \Omega(B)^{*}, \gamma \in \Omega(C)^{*}$ ( $\beta$ arbitrary). Suppose $a x=a^{\prime}$, $a^{\prime} x^{\prime}=a$ where $a=(b, c), a^{\prime}=\left(b^{\prime}, c^{\prime}\right)$ in terms of the direct decomposition, and $x, x^{\prime} \in \Omega(A)$ where $x^{\prime}$ has entries $\alpha^{\prime}, \beta^{\prime}, \gamma^{\prime}$ as in (1). Thus $c \gamma=c^{\prime}, c^{\prime} \gamma^{\prime}=c$ and, since $C$ is $P_{1}$, we may assume that $\gamma \in \Omega(C)^{*}$ and $\gamma^{\prime}=\gamma^{-1}$. Now $a y=a^{\prime}$ where $y=x+\left(1-x x^{\prime}\right) t$ and we wish to choose $t$ so that $y \in \Omega(A)^{*}$. Letting $t$ have entries $\lambda, \mu, \nu$ as in (1) we have

$$
y=\left(\begin{array}{ll}
\alpha+\left(1-\alpha \alpha^{\prime}\right) \lambda & 0 \\
\beta-\left(\beta \alpha^{\prime}+\gamma \beta^{\prime}\right) \lambda & \gamma
\end{array}\right)
$$


and we wish to find $\lambda$ so that $\alpha+\left(1-\alpha \alpha^{\prime}\right) \lambda \in \Omega(B)^{*}$. This is immediate from Proposition 1.

We state the next lemma in more generality than needed. Recall that

(1) $R$ is noetherian if and only if direct sums of injective modules are injective;

(2) $R$ is hereditary if and only if factors of injective modules are injective.

Now let $A$ be a module over the noetherian hereditary ring $R$ and $\left\{A_{i}\right\}$ the collection of injective submodules. Since $d A=\sum A_{i}$ is a factor of $\oplus A_{i}$ it is injective and is the unique largest injective submodule. Putting $A=d A \oplus r A$, we see that $r A$ is reduced (has no nonzero injective submodules) and is determined up to isomorphism by $A$.

LEMMA 7. Let $R$ be a noetherian hereditary ring and $C$ a collection of $R$-modules closed under direct sums. To show that each $A \in C$ is $P_{1}$ as $\Omega(A)$-module it is sufficient to show that each $r A$ is $P_{1}$ as $\Omega(r A)$-module.

Proof. If $a_{1}, a_{2} \in A$, where $a_{i}=\left(b_{i}, c_{i}\right)$ in terms of a decomposition $A=d A \oplus r A$, let $B$ be a submodule of $d A$ which is the injective hull of $b_{1} R+b_{2} R$. Then $A^{\prime}=$ $B \oplus r A$ is a direct summand of $A$. By Lemma 2 we wish to prove that $A^{\prime}$ is $P_{1}$ as $\Omega$-module. As in the proof of Proposition 11, $B$ is finite dimensional; hence $\Omega(B)$ is $\pi_{\infty}$ by Theorem 1, Corollary 3. If $\alpha \in \operatorname{Hom}_{R}(B, r A)$ then the image of $\alpha$, being a factor of $B$, is injective, and since $r A$ is reduced, $\alpha=0$. An application of Lemma 6 completes the argument.

Our principal motivation in this section was to extend Corollary 1 of Theorem 1 . For this reason and also to avoid undue complication, we state our main result (Theorem 3) for abelian groups, though a considerable portion of it carries over, with appropriate changes in terminology, to modules over dedekind domains. (Recall that a hereditary domain $R$ is the same thing as a dedekind domain, so a hereditary domain is automatically noetherian. These domains are characterized by the fact that a divisible module is the same thing as an injective module. Included is the case $R=Z$, so the previous lemma applies to abelian groups.)

First we discuss the classes of groups which occur in the theorem. (We sometimes say 'group' when it is clear that we mean 'abelian group'.) A selfinjective group is easily seen to be one with the following property: if the order of $a$ divides the order of $b$ (where the order of 0 is 1 , the order of a torsion-free element is $\infty$, all integers and $\infty$ divide $\infty$ ) then every integer dividing $b$ also divides $a$, i.e., $n x=b$ solvable implies $n x=a$ solvable. In fact we have the following (where a primary group is called homogeneous if it is the direct sum of arbitrarily many cyclic groups of the same order):

Structure Theorem. Let $A$ be an abelian group, $B$ its torsion subgroup and $B_{p}$ the primary components of $B$. Then $A$ is selfinjective if and only if either

(i) $B \neq A$ and $A$ is divisible, or

(ii) $B=A$ and each $B_{p}$ is either divisible or homogeneous. 
We do not stop to prove this here but only mention the following useful facts, valid for arbitrary $R$ :

Lemma 8. If $A$ is selfinjective and $I=\sum^{*} X_{i}$ a direct decomposition of the injective hull of $A$, then $A=\Sigma^{*}\left(A \cap X_{i}\right)$.

This follows easily from Lemma 3.

LEMma 9. A direct summand of a selfinjective module is selfinjective.

Note however that a direct sum of selfinjective modules need not be selfinjective; examples are immediately obtained from the above theorem.

The structure theorem also shows that selfinjective groups are included in the following much wider class:

We say that the abelian group $A$ is of type $T$ if every pair of elements in $r A$ is contained in a finitely generated direct summand of $r A$. This class is closed under arbitrary direct sums (because $d\left(\oplus A_{i}\right)=\oplus d A_{i}$, hence $\left.r\left(\oplus A_{i}\right)=\oplus r A_{i}\right)$ and includes

(1) sums of cyclic groups, for example finitely generated groups and free groups;

(2) selfinjective groups, for example divisible groups;

(3) periodic groups no primary component of which has elements of infinite height $\left({ }^{9}\right)$; and

(4) sundry other groups, for example complete products of copies of the infinite cyclic group.

TheOrem 3. The abelian group $A$ is $P_{\infty}$ as an $\operatorname{End}_{z} A$-module provided that $A$ is either

(i) of type $T$, or

(ii) periodic with every pair of elements contained in a countable direct summand.

Proof. (i) By Lemma 1 we wish to show that $A$ is $P_{1}$. By Lemmas 7 and 2 we may assume that $A$ is finitely generated, say $A=B \oplus C$ where $B$ is finite and $C$ is free. By Theorem $1 \Omega(B)$ is $\pi_{1}$ and by Lemma 5 and Theorem 2, $C$ is $P_{1}$ as $\Omega(C)$ module. Lemma 6 completes the argument. (The case when $A$ is selfinjective is also covered by Proposition 11.)

(ii) By Lemmas 1 and 2 we wish to show that a countable periodic group $A$ is $P_{1}$. By Lemma 4 we may assume that $A$ is primary and by Lemma 7 that $A$ is reduced. If $a$ and $b$ are elements sent onto each other by endomorphisms then $a \rightarrow b$ defines a height preserving isomorphism $\eta$ from the subgroup $a Z$ to the subgroup $b Z$. From the proof of Ulm's theorem as given in Kaplansky's book, Infinite Abelian groups, one knows that $\eta$ is extendible to an automorphism of $A$, as required.

( ${ }^{9}$ L. Fuchs pointed out to me in conversation that such a group is of type $T$. When countable, such a group is a sum of cyclic groups, but not in general. 
6. Further examples. Given a ring $R$ and an integer $n$ in general it seems to be a very difficult problem to obtain a catalogue of all $P_{n}$-modules. Let us consider what is perhaps the simplest nontrivial case: $R=Z, n=1$.

Proposition 12. The abelian group $A$ is $P_{1}$ as a Z-module if and only if its torsion subgroup $t A$ has exponent 1, 2, 3, 4 or 6.

Proof. Suppose first that $t A$ has one of the exponents listed. (Exponent 1 means that $A$ is torsion-free and we already know by Theorem 2 that in this case $A$ is $P_{\infty}$.) Thus supppose that $a x=b, b y=a$ where $a, b \in A, x, y \in Z$, and neither $x$ nor $y$ is \pm 1 . Then the cyclic subgroup $a Z=b Z$, being annihilated by $1-x y \neq 0$ has order $1,2,3,4$ or 6 , and since $a$ and $b$ are both generators we have indeed $a= \pm b$.

For the converse we prove a more general statement $(C(m)$ denotes the cyclic group of order $m$ ): if $A$ contains $C(m)^{n}$ as a subgroup where $m=5$ or $m \geqq 7$, then $A$ is not $P_{n}$.

Proof. We represent the elements of $C(m)^{n}$ as $n$-tuples of integers $\bmod m$. Take $k \not \equiv \pm 1 \bmod m$ with $k$ relatively prime to $m$. Let $a_{i}=(0, \ldots, 1, \ldots 0) \in C(m)^{n}$ (with a single 1 in the $i$ th position) and let $b=(k, 0, \ldots, 0)$. Then the two $n$-tuples $\left(a_{1}, \ldots, a_{n}\right),\left(b, a_{2}, \ldots, a_{n}\right)$ both generate the subgroup $C(m)^{n}$ but are not invertibly related; for if $\left(a_{1}, \ldots, a_{n}\right) x=\left(b, a_{2}, \ldots, a_{n}\right)$ then det $x \equiv k \bmod m$ so that $x \notin Z_{(n)}^{*}$.

It seems likely that the converse of the above statement is true (so that the absence of subgroups of type $C(m)^{n}, m=5$ or $m \geqq 7$, is a necessary and sufficient condition for $A$ to be $P_{n}$ ); however a general proof appears to be complicated and uninviting. Let us merely observe the special case promised in the comment after Proposition 3: every cyclic group is $P_{n}$ as Z-module for all $n \geqq 2$.

Proof. The infinite cyclic group is covered by Theorem 2, so we are concerned with $C(m)$, the integers $\bmod m, m>0$. Since a subgroup of a cyclic group is cyclic it is sufficient to show that any $n$-tuple $\left(a_{1}, \ldots, a_{n}\right)$ of elements which generate the whole group $C(m)$ is invertibly related to the $n$-tuple $(1,0, \ldots, 0)$. If $\{x, \ldots, y\}$ denotes the greatest common divisor of the integers $x, \ldots, y$, we have $\left\{a_{1}, \ldots, a_{n}, m\right\}$ $=1$. It is well known, and easily proved, that since $n \geqq 2$ there exist $a_{i}^{\prime} \equiv a_{i} \bmod m$ such that $\left\{a_{1}^{\prime}, \ldots, a_{n}^{\prime}\right\}=1$. By the Corollary to Proposition $10,\left(a_{1}^{\prime}, \ldots, a_{n}^{\prime}\right)$ occurs as the first row of some $X \in Z_{(n)}^{*}$. Thus $(1,0, \ldots, 0) X \equiv\left(a_{1}, \ldots, a_{n}\right)$, as required.

If $R$ is an integral domain a prime of $R$ is an element $p \neq 0$ such that $p R$ is a prime ideal, that is, if $p$ divides a product then it divides one of the factors (which is more than saying that $p$ does not factor). We write $a \mid b$ for $b \in a R$, and $a \nmid b$ for $b \notin a R$.

Proposition 13. If $R$ is a $P_{2}$ integral domain, and $p, a, b, c$ elements of $R$ such that $p$ is prime, $p \nmid a, a \mid p b$ and $c \mid(1-b)$, then there exist $s, t \in R$ such that $c+a s$ $+p t \in R^{*}$. 
Proof. Let $a r=p b, c d=1-b$. Then $a R+p R=a R+p c R$ since $p=a r+p c d$. Thus there exists

$$
\left(\begin{array}{ll}
\alpha & \beta \\
\gamma & \delta
\end{array}\right) \in R_{(2)}^{*} \quad \text { such that }(a, p)\left(\begin{array}{ll}
\alpha & \beta \\
\gamma & \delta
\end{array}\right)=(a, p c) .
$$

Now $a \alpha+p \gamma=a$, whence $\alpha \equiv 1 \bmod p$; and $a \beta+p \delta=p c$, whence $\beta \equiv 0 \bmod p$, say $\beta=-p s$, so $\delta=c+a s$. Thus the unit

$$
u=\left|\begin{array}{ll}
\alpha & \beta \\
\gamma & \delta
\end{array}\right| \equiv\left|\begin{array}{cc}
1 & 0 \\
\gamma & c+a s
\end{array}\right| \equiv c+a s \quad \bmod p,
$$

and the result follows.

For example, $R=Z[x]$ is not $P_{2}$ : take $p=x, a=b=5, c=2$. (The pairs $(5, x)$ and $(5,2 x)$ generate the same ideal but are not invertibly related.) $R$ being an integral domain is trivially $P_{1}$; (but $R$ is not $\pi_{1}$ by example 5 of $\S 3$ ).

Thus $R$ is an example showing that

(1) a $P_{1}$ module need not be $P_{2}$;

(2) the assumption that $R$ is dedekind cannot be dropped from Theorem 2;

(3) if a ring $R$ is $P_{1}$ the same is not necessarily true of $R_{(n)}$. (For if $R_{(n)}$ is $P_{1}$ then the submodule $R^{n}$ is also $P_{1}$ as $R_{(n)}$-module, hence $R$ is $P_{n}$.) This problem was raised in [4, p. 466].

In general $\pi_{1}$ integral domains, say with $\Re(R)=0$ to avoid semilocal domains, appear to be quite scarce, although it seems difficult to obtain criteria of wide applicability. We shall derive one rather weak result in this connection.

First we give another formulation of the $\pi_{1}$ property, valid for arbitrary $R$. If $a \in R$ let $a^{\#}$ denote the set of right ideals maximal with respect to being disjoint from $\left\{1-a u: u \in R^{*}\right\}$ (note $a^{\#}=\varnothing$ if and only if $a \in R^{*}$ ).

Proposition 14. $R$ is $\pi_{1}$ if and only if for each $a$ and for each $J \in a^{\#}$ we have $a \in J$; and when this is so, each such $J$ is a maximal right ideal.

Proof. Suppose $J \in a^{\#}, a \notin J$. Then $a x+j=1-a u$ for some $x \in R, j \in J, u \in R^{*}$. Thus $a(x+u) \equiv 1 \bmod J$ but $a v \not \equiv 1 \bmod J$ for all $v \in R^{*}$; hence $R / J$ is not $P_{1}$. Conversely if $R$ is not $\pi_{1}$ we have $a x \equiv 1 \bmod I$ for some right ideal $I$ which does not meet the set $\left\{1-a u: u \in R^{*}\right\}$; we may choose a $J \in a^{\#}$ containing $I$. Since $a x \equiv 1 \bmod J, a \notin J$.

Finally suppose that $R$ is $\pi_{1}$ and $J \in a^{\#}$. If $b \notin J$ we have, with a selfexplanatory notation, $1-a u=j+b x$. Since $a \in J, 1 \in J+b R$ whence $J$ is a maximal right ideal.

Proposition 15. If $R$ is an integral domain, not a field, with $\Re(R)=0$ and $R^{*}$ finite, then $R$ is not $\pi_{1}$.

Proof. We suppose that $R$ is $\pi_{1}$ and derive a contradiction. Let $R^{*}=\left\{u_{1}, \ldots, u_{n}\right\}$ and choose $a \neq 0, a \notin R^{*}$. The elements of $a^{\#}$ are the maximal ideals $M_{i}$ which do not contain any of the elements $1-a u_{i}$; the latter elements are all nonzero and $a$ is contained in each $M_{i}$. The contradiction arises from the following fact. 
LEMma. Let $r_{1}, \ldots, r_{n}$ be nonzero divisors in the commutative ring $R$ and suppose $\Re(R)=0$. Then the intersection of the maximal ideals which do not contain any of the $r_{i}$ is 0 .

Proof. Denote the maximal ideals not containing any of the $r_{i}$ by $M$, and denote the remaining by $N$. If $x \in \bigcap M$ then $x r_{1} \cdots r_{n} \in \bigcap M$; also $x r_{1} \cdots r_{n} \in \bigcap N$ since each $N$ contains some $r_{i}$. Hence $x r_{1} \cdots r_{n} \in \Re(R)=0$, and since the $r_{i}$ do not divide 0 , $x=0$.

We now prove the results promised in example $6, \S 3$.

Proposition 16. If $K$ is an extension field of the rational field $Q, K: Q=n<\infty$, and $R$ is the integral closure of $Z$ in $K$, then $R$ is not $\pi_{1}$.

Proof. Let $p$ be a prime $>2 n+1, x$ a primitive root $\bmod p$, and $1=x y+p t, y$, $t \in Z$. If $R$ were $\pi_{1}$ there would exist $r \in R, u \in R^{*}$ such that $u=x+p t r$. Taking the norm $N_{K \mid Q}$ gives $\pm 1=x^{n}+p b$ for some $b \in Z$. But this is impossible since $x$ is a primitive root $\bmod p$ and $n<(p-1) / 2$.

If $R$ is a commutative ring and $x \in R$, let $\hat{x}: R \rightarrow R / x R$ denote the canonical map. Thus $\hat{x} R^{*}$ is a subgroup of $(R / x R)^{*}$ and we put $H_{x}=(R / x R)^{*} / \hat{x} R^{*}$. We call $R$ residually periodic if it satisfies either of the following equivalent conditions:

1. for each $x \in R$ the group $H_{x}$ is periodic;

2. for each pair $x, y \in R$ there exists $r \in R$ and $n \geqq 1$ such that $x^{n}+(1-x y) r \in R^{*}$.

We omit the simple proof of the equivalence of these two statements. Clearly this notion is a weakening of the $\pi_{1}$ property; in fact $R$ is $\pi_{1}$ if and only if each $H_{x}=1$.

If $R$ and $S$ are integral domains, $S$ is a finite integral extension of $R$ if it is of the form $R\left[s_{1}, \ldots, s_{n}\right]$ where each $s_{i}$ is integrally dependent on $R$.

Proposition 17. Let $R$ be an integral domain. If $R$ is residually periodic then for every pair $x, y \in R$ there exist a finite integral extension $S$ of $R$ and $s \in S$ such that $x+(1-x y) s \in S^{*}$. The converse holds if $R$ is integrally closed.

Proof. First let $R$ be residually periodic and put $z=1-x y$. Now

$$
\begin{aligned}
z R=z R R^{n-1} & =z R(x R+z R)^{n-1} \\
& =x^{n-1} z R+x^{n-2} z^{2} R+\cdots+z^{n} R .
\end{aligned}
$$

Since $v-x^{n}=z r$ for some $v \in R^{*}, r \in R$ we have

$$
v-x^{n}=x^{n-1} z a_{n-1}+\cdots+z^{n} a_{0}
$$

for some $a_{i} \in R$. Let $s$ be a root of the equation $\theta^{n}-\theta^{n-1} a_{n-1}+\cdots+(-1)^{n} a_{0}=0$ and put $u=x+z s$. Then $u$ is a root of $(\theta-x)^{n}-z(\theta-x)^{n-1} a_{n-1}+\cdots=\theta^{n}+\cdots$ $+(-1)^{n} v=0$, so $u^{-1}$ is also integral over $R . S=R\left[s, u^{-1}\right]$ is the required integral extension.

Conversely, to prove that $R$ is residually periodic let $x, y \in R, z=1-x y, s \in S$ and $u=x+z s \in S^{*}$. If $K$ and $L$ are the quotient fields of $R$ and $S$ and $L: K=n$, 
applying the norm $N_{L \mid K}$ gives $v=x^{n}+z r \in R^{*}$ for some $r \in R$. (We need $R$ integrally closed to be sure that $v \in R$.)

\section{COROLlARY 1. The ring of all algebraic integers is $\pi_{1}$.}

Proof. If $a, b$ are algebraic integers apply the proposition to $R=Z[a, b]$ which is residually periodic (indeed each $H_{x}$ is finite).

Similarly,

COROLlaRY 2. If $F$ is a finite field, and $x$ an indeterminate, then the integral closure of $F[x]$ in the algebraic closure of $F(x)$ is $\pi_{1}$.

There seems to be no reason to doubt that the rings of the two corollaries are $\pi_{\infty}$; but the calculations necessary for a proof appear to be quite complicated.

In view of these results one might be tempted to conjecture that the integral domain $R$ is $\pi_{1}$ if $R$ is integrally closed and its quotient field is algebraically closed. However this is refuted by the following example. Let $F$ be a field of characteristic 0 and let $R$ be the integral closure of $F[x]$ in an algebraic closure of $F(x)$. If $R$ were $\pi_{1}$ there would exist $r \in R$ such that $(1-x)+x^{2} r=u \in R^{*}$. Applying the norm $N_{F(x, r) \mid F(x)}$ to this equation gives $(1-x)^{n}+x^{2} y \in F[x]^{*}=F^{*}$, where $n=F(x, r): F(x)$ and $y \in F[x]$. But this is impossible since the term $-n x$ cannot be cancelled.

We should mention that when $R$ is integrally closed every finite integral extension $S$ in which one has $x+(1-x y) s \in S^{*}$ is obtained by the method given in the proof. For let $K$ and $L$ be the quotient fields of $R$ and $S$ and let $s$ have the field polynomial $\theta^{n}-\theta^{n-1} a_{n-1}+\cdots+(-1)^{n} a_{0}$ with respect to $L \mid K$. Then the constant term of the field polynomial for $u=x+z s$ is $x^{n}+x^{n-1} z a_{n-1}+\cdots+z^{n} a_{0}=v$ which must be a unit since $u$ is a unit and $R$ is integrally closed. The procedure of the proof obviously gives back $u=x+z s$.

As an example let us take $R=Z, x=2, y=3$ and determine all quadratic fields with the desired property. If $s$ satisfies $\theta^{2}-\theta a_{1}+a_{0}=0$ then $u=x+z s=2-5 s$ satisfies $\theta^{2}+\left(5 a_{1}-4\right) \theta+\left(25 a_{0}-10 a_{1}+4\right)=0$. Since $u$ is a unit we have $25 a_{0}-10 a_{1}$ $+4=-1\left(+1\right.$ is impossible), the general solution of which is $a_{0}=1+2 t, a_{1}=3+5 t$, $t \in Z$. Hence $s=\left(3+5 t+\left(5+22 t+25 t^{2}\right)^{1 / 2}\right) / 2$. The quadratic is positive definite and the fields are $Q(\sqrt{ } 2), Q(\sqrt{ } 5), Q(\sqrt{ } 13), \ldots$ Similarly one can parameterize the quartic, sextic, . . f fields which 'split' the pair $(x, y)=(2,3)$ (there are no cubic, quintic, ... fields with this property).

APPENDIX. In this appendix we prove two results about the endomorphism ring of a module which are needed in the main part of the paper. We have put them in an appendix since they are of a general nature and not solely concerned with the Schröder-Bernstein problem.

If $A$ and $B$ are $R$-modules we define the radical $\Re\left(\operatorname{Hom}_{R}(A, B)\right)$ of the group $\operatorname{Hom}_{R}(A, B)$ to be the subgroup consisting of those $\alpha \in \operatorname{Hom}_{R}(A, B)$ such that for all $\beta \in \operatorname{Hom}_{R}(B, A)$ we have $\alpha \beta \in \Re\left(\operatorname{End}_{R} A\right)$, where in the latter case $\Re$ denotes 
the Jacobson radical of the ring. Clearly $\Re\left(\operatorname{Hom}_{R}(A, A)\right)=\Re\left(\operatorname{End}_{R} A\right)$, so the notation is consistent $\left({ }^{10}\right)$.

Now let $A_{1}, \ldots, A_{n}$ be $R$-modules, $A=A_{1} \oplus \cdots \oplus A_{n}$ and $S=\operatorname{End}_{R} A$, so the elements of $S$ are $n$ by $n$ matrices $\left(\alpha_{i j}\right)$ where $\alpha_{i j} \in \operatorname{Hom}_{R}\left(A_{i}, A_{j}\right)=H_{\imath j}$.

Proposition A. With the above notation,

$$
\Re(S)=\left\{\left(\alpha_{i j}\right): \text { for all } i, j, \alpha_{i j} \in \mathfrak{R}\left(H_{i j}\right)\right\} .
$$

Proof. Let $J$ denote the right hand side of the equation. Since $\Re\left(H_{i j}\right)$ is a subgroup of $H_{i j}, J$ is closed under addition. If $\left(\alpha_{i j}\right) \in J$ and $\left(\beta_{i j}\right)$ is arbitrary let $\left(\gamma_{i j}\right)=$ $\left(\alpha_{i j}\right)\left(\beta_{i j}\right)$ so $\gamma_{i j}=\sum \alpha_{i k} \beta_{k j}$. Now if $\delta_{j i} \in H_{j i}$ then $\alpha_{i k} \beta_{k j} \delta_{j i}=\alpha_{i k} \eta_{k i}$ where $\eta_{k i} \in H_{k i}$, and since $\alpha_{i k} \in \Re\left(H_{i k}\right), \alpha_{i k} \beta_{k j} \delta_{j i} \in \Re\left(H_{i i}\right)$, so $J$ is a right ideal. It follows that $J_{i}$, the set of $\alpha \in J$ whose rows other than the $i$ th are 0 , is a right ideal. If $\alpha \in J_{1}$ then $1+\alpha$ is the identity matrix except that its first row is $\left(1+\alpha_{11}, \alpha_{12}, \ldots, \alpha_{1 n}\right)$. Since $\alpha_{11} \in \Re\left(H_{11}\right), \beta=1+\alpha_{11}$ is a unit and therefore $1+\alpha$ has as inverse the matrix whose first row is $\left(\beta^{-1},-\beta^{-1} \alpha_{12}, \ldots,-\beta^{-1} \alpha_{1 n}\right)$ and which otherwise coincides with the identity matrix. It follows that $J_{1} \subseteq \Re(S)$ and similarly $J_{i} \subseteq \Re(S)$. Hence $J \subseteq \Re(S)$.

Conversely let $\left(\alpha_{i j}\right) \in \mathfrak{R}(S)$ and let $\beta$ be the matrix all of whose entries are 0 except for $\beta_{p p}=1$, and $\gamma$ similarly all 0 except for $\gamma_{q p}$. Then $\delta=\beta \alpha \gamma$ has 0 entries except for $\delta_{p p}=\alpha_{p q} \gamma_{q p}$. Since $1+\delta$ is invertible so is $1+\delta_{p p}$, and since $\gamma_{q p}$ is an arbitrary element of $H_{q p}$ it follows that $\delta_{p p} \in \mathfrak{R}\left(H_{p p}\right)$, whence $\alpha_{p q} \in \Re\left(H_{p q}\right)$.

Corollary 1. If $\alpha \in \Re\left(\operatorname{Hom}_{R}(A, B)\right)$ and $\beta \in \operatorname{Hom}_{R}(B, A)$ then $\beta \alpha \in \Re\left(\operatorname{End}_{R} B\right)$ (hence $\Re\left(\operatorname{Hom}_{R}(A, B)\right)$ can be given a symmetrical definition).

The radical of a ring is an ideal and the corollary follows by applying the proposition to the module $A \oplus B$.

CoRollaRY 2. $\Re\left(R_{(n)}\right)=\Re(R)_{(n)}$.

This is the case $A=R^{n}$. (Strictly speaking $A=\left(R^{\circ}\right)^{n}$ where $R^{\circ}$ is the opposite ring of $R$, since we are writing the endomorphisms on the right.)

Proposition B. Let $R$ be a ring, $A$ an $R$-module of finite length, $S=\operatorname{End}_{R} A$ and $J=\Re(S)$. Then $J$ is nilpotent and $S / J$ is artinian semisimple.

Proof. We write $A=A_{1} \oplus \cdots \oplus A_{n}$ where the $A_{i}$ are indecomposable and apply Proposition A. Thus the elements of $S / J$ are $n$ by $n$ matrices $\bar{\alpha}$ with entries $\bar{\alpha}_{i j} \in \bar{H}_{i j}$ $=H_{i j} / \Re\left(H_{i j}\right)$. If $I_{i}$ denotes the right ideal of those $\bar{\alpha}$ whose rows other than the $i$ th are 0 , then $S / J=I_{1}+\cdots+I_{n}$. If $\bar{\alpha} \in I_{1}$ and $\bar{\alpha}_{1 p} \neq 0$, and $\bar{\beta}$ denotes the matrix with $\bar{\beta}_{p p}=1$ and 0 's elsewhere, then $\bar{\alpha} \bar{\beta}$ has the single nonzero entry $\bar{\alpha}_{1 p}$. By definition of $\Re\left(H_{1 p}\right)$, there exists $\bar{\delta}_{p 1} \in \bar{H}_{p 1}$ such that $\bar{\alpha}_{1 p} \bar{\delta}_{p 1} \neq 0$. Taking $\bar{\delta}$ to have the entry $\bar{\delta}_{p 1}$

${ }^{\left({ }^{10}\right)}$ If $R$ is commutative, $\operatorname{Hom}_{R}(A, B)$ is an $R$-module and therefore has a radical in the conventional sense, which is not to be confused with our $\Re$. 
and 0's elsewhere, we see that $\bar{\alpha} \bar{\beta} \delta$ has a single nonzero entry in $\bar{H}_{11}$. But $\bar{H}_{11}$ is a skew-field [1, p. 23]; hence $\bar{\alpha} \bar{\beta} \bar{\delta}$, and therefore $\bar{\alpha}$, generates the right ideal $I_{1}$. Thus $I_{1}$, and similarly $I_{i}$ is a minimal right ideal, and $S / J$ is artinian semisimple.

To show that $J$ is nilpotent (a fact not used in the paper) it suffices by $[1$, p. 26 , exercise 3] to prove that each element $\alpha \in J$ is nilpotent. (Then $J^{d}=0$ where $d$ is the length of $A$.) The sequence $A \alpha \supseteq A \alpha^{2} \supseteq \cdots$ becomes stationary, say $A \alpha^{r}=A \alpha^{r+1}$ $=\cdots$. Put $\beta=\alpha^{r}, B=A \beta, C=\operatorname{Ker} \beta$. The standard argument [1, p. 23] shows that $A=B \oplus C$ and $\gamma=\beta \mid B$ is an automorphism. We have $\delta=\beta\left(\gamma^{-1} \oplus 1_{C}\right) \in J$ where $\delta$ is idempotent (being the canonical projection $A \rightarrow B$ ). The radical contains no nontrivial idempotents, so $\delta=0$, and since $\gamma^{-1} \oplus 1$ is a unit, $\beta=0$, which completes the proof.

\section{REFERENCES}

1. N. Bourbaki, Algèbre, Chapitre 8, Hermann, Paris, 1958.

2. N. Jacobson, Structure of rings, Amer. Math. Soc. Colloq. Publ., Vol. 37, Amer. Math. Soc., Providence, R. I., 1956.

3. R. E. Johnson and E. T. Wong, Quasi-injective modules and irreducible rings, J. London Math. Soc. 36 (1961), 260-268.

4. I. Kaplansky, Elementary divisors and modules, Trans. Amer. Math. Soc. 66 (1949), 464-491.

5. J. Lambek, Lectures on rings and modules, Blaisdell, New York, 1966.

6. E. Steinitz, Rechteckige Systeme und Moduln in algebraischen Zahlkörpern I, Math. Ann. 71 (1912), 328-354; II, ibid. 72 (1912), 297-345.

7. E. T. Wong and R. E. Johnson, Selfinjective rings, Canad. Math. Bull. 2 (1959), 167-173.

8. D. Zelinsky, Linearly compact modules and rings, Amer. J. Math. 75 (1953), 79-90.

MCGILL UNIVERSITY, Montreal, Canada 